\title{
Kink ratchet induced by a time-dependent symmetric field potential
}

\author{
Bernardo Sánchez-Rey* \\ Departamento de Física Aplicada I, E.P.S., Universidad de Sevilla, Virgen de África 7, 41011 Sevilla, Spain \\ Jesús Casado-Pascual ${ }^{\dagger}$ \\ Física Teórica, Universidad de Sevilla, Apartado de Correos 1065, 41080 Sevilla, Spain \\ Niurka R. Quintero $\ddagger$ \\ Departamento de Física Aplicada I, E.P.S., Universidad de Sevilla, Virgen de África 7, 41011 Sevilla, Spain
}

(Received 28 April 2016; published 22 July 2016)

\begin{abstract}
The ratchet effect of a sine-Gordon kink is investigated in the absence of any external force while the symmetry of the field potential at every time instant is maintained. The directed motion appears by a time shift of the sine-Gordon potential through a time-dependent additional phase. A symmetry analysis provides the necessary conditions for the existence of net motion. It is also shown analytically, by using a collective coordinate theory, that the novel physical mechanism responsible for the appearance of the ratchet effect is the coupled dynamics of the kink width with the background field. Biharmonic and dichotomic periodic variations of the additional phase of the sine-Gordon potential are considered. The predictions established by the symmetry analysis and the collective coordinate theory are verified by means of numerical simulations. Inversion and maximization of the resulting current as a function of the system parameters are investigated.
\end{abstract}

DOI: 10.1103/PhysRevE.94.012221

\section{INTRODUCTION}

Solitons are localized nonlinear waves that behave like particles in many situations, with their own mass, velocity, and other particlelike properties [1]. By using a collective coordinate theory, it is shown that the soliton dynamics can be reduced to the study of a system of ordinary differential equations for collective variables, such as the kink center of mass, its width, etc. This scheme captures and explains the main features of different phenomena, such as soliton scattering and soliton diffusion [2].

An interesting phenomenon which appears in particle as well as in extended systems is the so-called ratchet effect, where particles or solitons manifest a unidirectional motion, generally due to the action of periodic forces of zero mean. In fact, in a similar way to that of the rectification of random motion of Brownian particles in periodic potentials [3], unidirectional motion of solitons is induced by breaking the spatiotemporal and/or field symmetries of the extended system [4,5]. Furthermore, characteristic features of the ratchet phenomena in point-particle systems also arise in the case of solitons. For instance, current reversals and resonance behaviors of the soliton average velocity are achieved through parameter variations of potentials, forces, and damping [4,6-8].

The relevance of these phenomena covers a wide range of areas from biophysics [9] to possible technological applications [10]. Specifically, soliton ratchets have been observed experimentally in the damped Josephson junctions driven by external asymmetric forces [11,12]. In these experiments, a long quasi-one-dimensional Josephson junction is described

\footnotetext{
*bernardo@us.es

†jcasado@us.es

†niurka@us.es
}

by the perturbed sine-Gordon equation

$$
\Phi_{t t}(x, t)-\Phi_{x x}(x, t)+U^{\prime}[\Phi(x, t)]=-\beta \Phi_{t}(x, t)+f(x, t)
$$

for the superconducting phase difference $\Phi(x, t)$ across the junction, where $\Phi_{x} \equiv \partial \Phi / \partial x, \Phi_{t} \equiv \partial \Phi / \partial t, \beta>0$ is the damping coefficient, $U^{\prime}(z)$ is the derivative with respect to $z$ of a cosine potential $U(z)$, and $f(x, t)$ is an external force. In this system, the ratchet effect of kink (or antikink) excitations is induced: (i) by using a symmetric field potential, $U(\Phi)=1-\cos (\Phi)$, together with an external periodic force that breaks either temporal symmetries $[4,7,11,13]$ or spatial symmetries [14]; (ii) by using an asymmetric sawtooth potential of the type $U(\Phi)=C-\cos (\Phi)+(\lambda / 2) \sin (2 \Phi)$, where $C$ and $\lambda$ are constants, plus an external ac force $f(t)[6,8,14,15]$; (iii) by considering local and periodic arrays of inhomogeneities $U(\Phi, x)=1-\cos (\Phi)\left[1+\epsilon \sum_{i, n} \delta(x-\right.$ $\left.x_{i}-n L\right)$ ] (microshorts along the Josephson junctions), together with the action of an ac force $f(t)[16,17]$; and finally, (iv) by modulating the field potential with an ac force, $U(\Phi, t)=1-\cos (\Phi)\left[1+\epsilon_{1} \sin \left(\omega_{1} t\right)\right]$, together with an additive ac signal $f(t)=\epsilon_{2} \sin \left(\omega_{2} t\right)$ [18].

It is interesting to note that in all the cases mentioned above, the ratchet mechanism is due to a combination of a periodic potential with space- or time-dependent external forces. However, for an ensemble of Brownian particles, a directed current has also been obtained solely by using a symmetric periodic potential that alternates between two states that differ only by a discrete translation [19]. It is therefore natural to pose the following question: Can a directed motion of kinks be obtained in the absence of any external force while keeping the field potential symmetry at every time instant? The aim of this paper is to answer this question by extending this ratchet mechanism to the sine-Gordon kink. The key idea is to shift the sine-Gordon potential forwards and backwards by 
introducing a time-dependent additional phase. In contrast to the Brownian particle case, net motion of kink is achieved in the absence of noise. Here a novel mechanism of soliton ratchets appears, where, unlike other models, the background field plays a decisive role in the generation of net motion.

The outline of the paper is as follows. A full description of the model under consideration is presented in Sec. II. Necessary conditions for the occurrence of net motion are established by using a symmetry analysis. In Sec. III, a collective coordinate approach is developed in order to provide a physical insight into the ratchet mechanism. In Sec. IV, the theoretical results of the previous sections are then compared with numerical simulations. Biharmonic and dichotomic periodic signals are used to shift the sine-Gordon potential in time and the dependence of the ratchet velocity on the system parameters is investigated. Finally, the main contributions of our work are summarized in the last section.

\section{DESCRIPTION OF THE MODEL AND SYMMETRY ANALYSIS}

In this study, our attention is focused on a sine-Gordon system of the form

$$
\Phi_{t t}(x, t)-\Phi_{x x}(x, t)+\beta \Phi_{t}(x, t)+U^{\prime}[\Phi(x, t), t]=0
$$

with a time-dependent potential

$$
U(\Phi, t)=1-\cos [\Phi+\theta \eta(t)],
$$

where $\eta(t)$ is a periodic function of period $T$ and zero timeaverage (such that $\int_{0}^{T} d t \eta(t) / T=0$ ), and $\theta$ is a parameter introduced to adjust the amplitude of $\eta(t)$. For a fixed value of $\theta \eta(t)=\kappa$, the potential considered above corresponds to that used to model what is called in the literature a $\kappa$ junction [20], that is, a Josephson junction with an additional phase shift $\kappa$. Our model is inspired by experimental observations of transitions from positive ("0-phase state") to negative (" $\pi$-phase state") coupling between the superconductors of a junction with a graphene interlayer, which can easily be controlled by a gate voltage [21]. Similar transitions, induced, for instance, by temperature variations, have also been observed with a ferromagnetic interlayer [22]. The potential (3) can be considered a theoretical generalization of such observations.

To fully specify the mathematical problem, the partial differential equation (2)-(3) must be amended by both initial and boundary conditions. Since we are interested in studying solutions of Eqs. (2)-(3) with only one kinklike structure present and, consequently, with topological charge $2 \pi$, we consider aperiodic boundary conditions of the form [23]

$$
\begin{gathered}
\lim _{x \rightarrow+\infty} \Phi(x, t)=\lim _{x \rightarrow-\infty} \Phi(x, t)+2 \pi, \\
\lim _{x \rightarrow+\infty} \Phi_{x}(x, t)=\lim _{x \rightarrow-\infty} \Phi_{x}(x, t) .
\end{gathered}
$$

Additionally, the following initial conditions at time $t_{0}$ are assumed:

$$
\begin{gathered}
\Phi\left(x, t_{0}\right)=4 \arctan \left(e^{x}\right), \\
\Phi_{t}\left(x, t_{0}\right)=0,
\end{gathered}
$$

which correspond to an unperturbed kink centered at $x=0$ and at rest.

The center of mass of the kink and its time-average velocity can be respectively calculated from the expressions

$$
X(t)=\frac{1}{2 \pi} \int_{-\infty}^{+\infty} d x \times \Phi_{x}(x, t)
$$

and

$$
V=\lim _{\Delta t \rightarrow \infty} \frac{1}{\Delta t} \int_{t_{0}}^{t_{0}+\Delta t} d t X_{t}(t)=\lim _{\Delta t \rightarrow \infty} \frac{X\left(t_{0}+\Delta t\right)}{\Delta t},
$$

where, according to Eq. (6), it has been used that $X\left(t_{0}\right)=0$.

Let us now examine the conditions under which a net motion of the kink may be expected to occur. To this end, let $\Phi\left(x, t ; \theta, t_{0}\right)$ be the solution of the problem defined by Eqs. (2)(7) where, for convenience, its dependence on the parameters $\theta$ and $t_{0}$ has been explicitly indicated. It is then straightforward to verify that the function $2 \pi-\Phi\left(-x, t ;-\theta, t_{0}\right)$ is also a solution of the same problem. Consequently, from the uniqueness of the solution of the problem (2)-(7), it follows that $\Phi\left(x, t ; \theta, t_{0}\right)=$ $2 \pi-\Phi\left(-x, t ;-\theta, t_{0}\right)$ and, taking into account Eqs. (8) and (9), that

$$
V\left(\theta, t_{0}\right)=-V\left(-\theta, t_{0}\right)
$$

Now let us assume that the periodic function $\eta(t)$ satisfies the following time-shift symmetry:

$$
\eta(t)=-\eta(t+T / 2) .
$$

In this case, it is easy to show that the function $\Phi(x, t+$ $\left.T / 2 ;-\theta, t_{0}+T / 2\right)$ is a solution of the problem (2)-(7). Thus, from the uniqueness of the solution, it follows that $\Phi\left(x, t ; \theta, t_{0}\right)=\Phi\left(x, t+T / 2 ;-\theta, t_{0}+T / 2\right)$ and, bearing in mind Eqs. (8) and (9), that

$$
V\left(\theta, t_{0}\right)=V\left(-\theta, t_{0}+T / 2\right)
$$

It can be seen that the time-average velocity is independent of the initial time $t_{0}$, i.e., $V\left(\theta, t_{0}\right)=V(\theta)$. Consequently, in order to generate a net motion, the symmetry (11) must be broken, since otherwise from Eqs. (10) and (12) it would follow that $V(\theta)=0$.

\section{COLLECTIVE COORDINATE APPROACH}

Physical insight into the appearance of net kink transport can be gained by means of a collective coordinate approach. To this end, let us define the "naked" kink field as $\Psi(x, t)=$ $\Phi(x, t)-\varphi(t)$, where $\varphi(t)=\lim _{x \rightarrow-\infty} \Phi(x, t)$ is the background field. This background field satisfies the differential equation

$$
\begin{aligned}
\varphi_{t t}(t) & =-\beta \varphi_{t}(t)-U^{\prime}[\varphi(t), t] \\
& =-\beta \varphi_{t}(t)-\sin [\varphi(t)+\theta \eta(t)],
\end{aligned}
$$

with the initial conditions $\varphi\left(t_{0}\right)=\varphi_{t}\left(t_{0}\right)=0$. 
The momentum and the energy of the "naked" kink are respectively given by the expressions

$$
P(t)=-\int_{-\infty}^{+\infty} d x \Psi_{t}(x, t) \Psi_{x}(x, t)
$$

and

$E(t)=\int_{-\infty}^{+\infty} d x\left\{\frac{\left[\Psi_{t}(x, t)\right]^{2}}{2}+\frac{\left[\Psi_{x}(x, t)\right]^{2}}{2}+\widetilde{U}[\Psi(x, t), t]\right\}$,

with $\widetilde{U}[\Psi(x, t), t]$ being the new potential $U[\Psi(x, t)+$ $\varphi(t), t]-U[\varphi(t), t]$. In order to obtain a finite result for $E(t)$, the zero of this new potential has been chosen so that $\lim _{x \rightarrow \pm \infty} \widetilde{U}[\Psi(x, t), t]=0$. By differentiating with respect to time Eqs. (14) and (15), and using Eqs. (2), (3), and (13), it is easy to show that

$$
P_{t}(t)=-\beta P(t)-2 \pi \sin [\varphi(t)+\theta \eta(t)]
$$

and

$$
\begin{aligned}
E_{t}(t)= & {\left[\varphi_{t}(t)+\theta \eta_{t}(t)\right] \int_{-\infty}^{+\infty} d x\{\sin [\Psi(x, t)+\varphi(t)+\theta \eta(t)]} \\
& -\sin [\varphi(t)+\theta \eta(t)]\}+\sin [\varphi(t)+\theta \eta(t)] \\
& \times \int_{-\infty}^{+\infty} d x \Psi_{t}(x, t)-\beta \int_{-\infty}^{+\infty} d x\left[\Psi_{t}(x, t)\right]^{2} .
\end{aligned}
$$

Let us now consider an ansatz for the "naked" kink field of the form

$$
\Psi^{(\mathrm{a})}(x, t)=4 \arctan \left\{\exp \left[\frac{x-X(t)}{L(t)}\right]\right\}
$$

where $X(t)$ and $L(t)$ are, respectively, the center of mass and the width of the kinklike structure.

By inserting this ansatz into Eqs. (14) and (15), one obtains that

$$
P(t)=\frac{8 X_{t}(t)}{L(t)},
$$

where $8 / L(t)$ plays the role of an effective mass, and

$$
E(t)=\frac{\pi^{2}\left[L_{t}(t)\right]^{2}+12\left\{1+\left[X_{t}(t)\right]^{2}+[L(t)]^{2} \cos [\varphi(t)+\theta \eta(t)]\right\}}{3 L(t)} .
$$

From Eqs. (13) and (16), it is easy to see that the function $f(t)=P(t)-2 \pi \varphi_{t}(t)$ satisfies the differential equation $f_{t}(t)=-\beta f(t)$. In addition, from Eqs. (7) and (14), it is clear that $f\left(t_{0}\right)=0$, and hence $f(t)=0 \forall t \geqslant t_{0}$. Consequently, it is obtained that $P(t)=2 \pi \varphi_{t}(t)$. Thus, according to Eq. (19), the kink velocity can be expressed as

$$
X_{t}(t)=\frac{\pi L(t) \varphi_{t}(t)}{4} .
$$

Remarkably, this expression shows that a net motion of the kink appears due to the coupling between the background field and the width of the kink. In contrast to other soliton ratchet mechanisms, where the background field is used only to improve the collective coordinate theory [24], here $\varphi(t)$ plays an essential role in the ratchet effect.

A differential equation for the time evolution of $L(t)$ can be obtained by replacing Eqs. (18) and (20) in Eq. (17) and using Eq. (21). After lengthy calculations, one finds

$$
\begin{aligned}
L_{t t}(t)= & \frac{\left[L_{t}(t)\right]^{2}}{2 L(t)}-\frac{3 L(t)\left[\varphi_{t}(t)\right]^{2}}{8}-\beta L_{t}(t) \\
& +\frac{6}{\pi^{2} L(t)}\left\{1-[L(t)]^{2} \cos [\varphi(t)+\theta \eta(t)]\right\},
\end{aligned}
$$

which has to be solved with the initial conditions $L\left(t_{0}\right)=1$ and $L_{t}\left(t_{0}\right)=0$. The time-average velocity can be calculated from Eqs. (9) and (21) after numerically solving the differential equations (13) and (22).

A further simplification of the collective coordinate approach can be obtained by linearizing the nonlinear differential equations (13) and (22). To this end, let $\sum_{n=0}^{\infty} \theta^{n} \varphi^{(n)}(t) / n$ ! and $\sum_{n=0}^{\infty} \theta^{n} L^{(n)}(t) / n$ ! be the power expansion in $\theta$ of the background field and the kink width, respectively. It is then easy to show that $\varphi^{(1)}(t)$ and $L^{(2)}(t)$ satisfy the linear differential equations

$$
\varphi_{t t}^{(1)}(t)+\beta \varphi_{t}^{(1)}(t)+\varphi^{(1)}(t)=-\eta(t)
$$

and

$$
\begin{aligned}
& L_{t t}^{(2)}(t)+\beta L_{t}^{(2)}(t)+\frac{12}{\pi^{2}} L^{(2)}(t) \\
& \quad=\frac{6}{\pi^{2}}\left[\varphi^{(1)}(t)+\eta(t)\right]^{2}-\frac{3}{4}\left[\varphi_{t}^{(1)}(t)\right]^{2},
\end{aligned}
$$

and that $\varphi^{(0)}(t)=L^{(1)}(t)=0$ and $L^{(0)}(t)=1$. According to Eqs. (23) and (24), it is clear that after a transient time $\varphi^{(1)}(t)$ and $L^{(2)}(t)$ become periodic functions of $t$ with period $T$. Therefore, from Eqs. (9) and (21) one can see that the timeaverage velocity is approximately given by the expression

$$
V \approx \frac{\pi \theta^{3}}{8 T} \int_{0}^{T} d t \tilde{\varphi}_{t}^{(1)}(t) \tilde{L}^{(2)}(t),
$$

where $\tilde{\varphi}^{(1)}(t)$ and $\tilde{L}^{(2)}(t)$ are, respectively, the periodic solutions of Eqs. (23) and (24).

\section{NUMERICAL SIMULATIONS}

In order to check the existence of net kink motion when symmetry conditions are broken, we have performed numerical simulations of the damped sine-Gordon equation (2)-(3) for two particular choices of the function $\eta(t)$. The initial and boundary conditions are given by Eqs. (6)-(7) and (4)-(5), respectively. The algorithm used is a Runge-Kutta-Verner fifth-order method with space step $\Delta x=0.02$ and adaptive step size in time. 

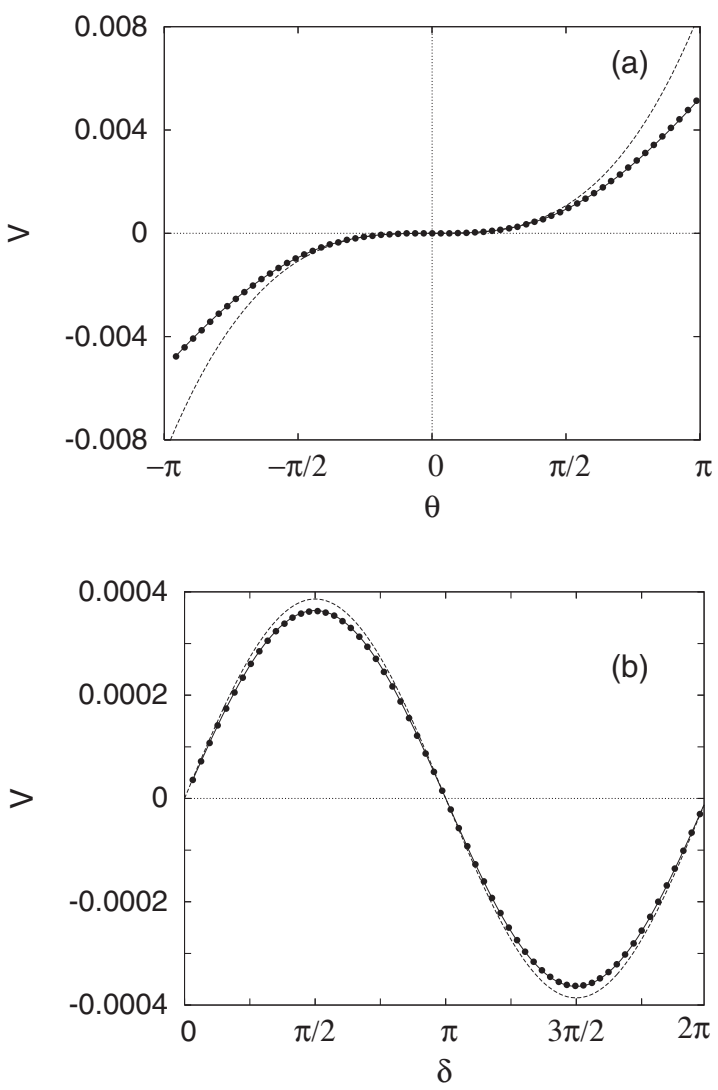

FIG. 1. (a) Kink velocity versus the amplitude parameter $\theta$ for fixed $\delta=0.8$. The circles are the results obtained by simulation of the sine-Gordon equation (2)-(3), the solid line represents the average velocity obtained by using the collective coordinate Eq. (21), and the dashed line corresponds to its linear approximation (25). (b) Kink velocity versus the phase difference $\delta$ for fixed $\theta=1$. In both panels, $\beta=0.1$ and $\omega=0.1$.

\section{A. Biharmonic case}

For a first numerical test, we have chosen the biharmonic function $\eta(t)=\cos (\omega t)+\cos (2 \omega t+\delta)$, since it is a prototypical periodic function that breaks the time-shift symmetry given by Eq. (11) [25,26].

In Fig. 1(a), the dependence of the average velocity on the amplitude $\theta$ is shown. The circles represent the simulation results while the solid line corresponds to the collective coordinate approach obtained from solving the differential equations (13) and (22). Notice the excellent agreement between the simulations and the collective coordinate approximation even for large values of $\theta$. With a dashed line, the average velocity obtained using the linear approximation (25) of the collective coordinate equations has also been plotted. As expected, the linear approximation goes well only for small values of the perturbation amplitude $\theta$. In this regime, $V \sim A \theta^{3}$, where $A$ is independent of $\theta$. This functional dependence on the perturbation amplitude has been proved to occur in a very general framework, independently of the system details, by using simple symmetry considerations [27,28]. The linear collective coordinate equations allow us to calculate the dependence on the rest of the parameters of the prefactor that multiplies the $\theta^{3}$ term.

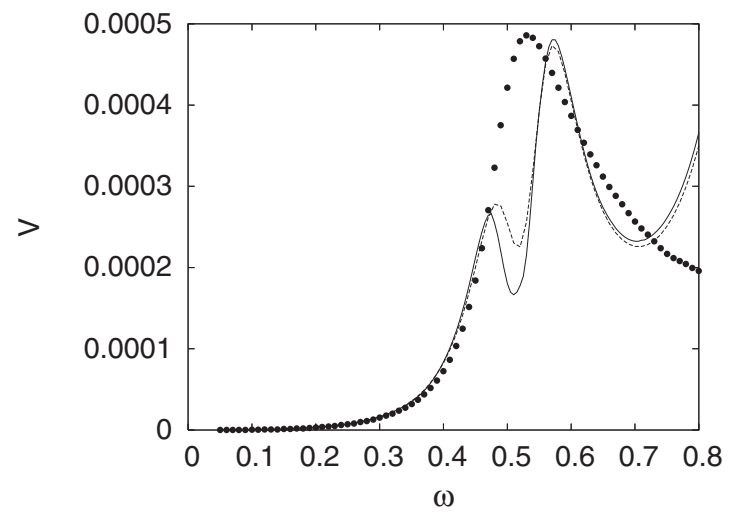

FIG. 2. Kink velocity versus frequency $\omega$ for fixed $\theta=0.1, \delta=$ 0.8 , and $\beta=0.2$. The circles are the results obtained by simulation of the sine-Gordon equation (2)-(3), the solid line represents the average velocity obtained by using the collective coordinate Eq. (21), and the dashed line corresponds to its linear approximation (25).

Equally, for sufficiently small perturbation amplitudes, the general formalism developed in Refs. [27] and [28] together with Eq. (10) lead to $V \sim B \cos \left(\delta+\delta_{0}\right)$, where $B$ and $\delta_{0}$ are independent of $\delta$. This dependence of $V$ on the phase difference $\delta$ is displayed in Fig. 1(b) for fixed $\theta=1$. For the chosen parameters, clearly $\delta_{0} \approx \pi / 2$. Once again the agreement between the collective coordinate theory (solid line) and the simulation results (circles) is excellent. The slight deviation of the linear collective coordinate approximation (dashed line) is due to the relatively large value of $\theta$ used.

Finally, in Fig. 2, the dependence of $V$ on the frequency $\omega$ is shown. The value of the amplitude employed, $\theta=0.1$, is rather small and for that reason the linear approximation (dashed line) closely matches the collective coordinate results (solid line). The collective coordinate theory fits the simulation results (circles) very well, but only for low frequencies. For frequencies $\omega \gtrsim 0.45$, significative discrepancies appear due to the fact that the frequency $\omega$ approaches $\omega_{p h}=1$ (the lowest frequency of the phonons) and therefore the phonons can become excited. Consequently, this outcome indicates that the collective coordinate theory has an "adiabatic" nature and its validity requires that perturbations must be applied in a sufficiently slow way [29].

\section{B. Dichotomic case}

In this section, $\eta(t)$ is a dichotomic periodic function of time that successively takes the values +1 and -1 during time intervals of lengths $\tau_{+1}$ and $\tau_{-1}$, respectively, thereby providing the period $T=\tau_{+1}+\tau_{-1}$. Therefore, according to Eq. (3), the time-dependent potential $U(\Phi, t)$ can only be in one of two possible states, $U_{+1}(\Phi)=1-\cos (\Phi+$ $\theta)$ or $U_{-1}(\Phi)=1-\cos (\Phi-\theta)$, which differ merely by a translation of $2 \theta$. It is not difficult to see that this function can be represented as

$$
\eta(t)=\operatorname{sgn}\left[\sin \left(\frac{\omega \Delta \tau}{4}\right)-\sin (\omega t)\right],
$$

where $\omega=2 \pi / T$ is the frequency and $\Delta \tau=\tau_{+1}-\tau_{-1} \in$ $[-T, T]$. 
The choice (26) introduces a new symmetry property not present in the biharmonic case. Indeed, let $\Phi\left(x, t ; \theta, \Delta \tau, t_{0}\right)$ be the solution of the problem defined by Eqs. (2)-(7) and (26), then $\Phi\left(x, t+T / 2 ;-\theta,-\Delta \tau, t_{0}+T / 2\right)$ is also solution of the same problem. Taking into account that the average velocity is independent of the initial time $t_{0}$, it follows that

$$
V(\theta, \Delta \tau)=V(-\theta,-\Delta \tau) .
$$

From Eqs. (10) and (27) one obtains

$$
V(\theta, \Delta \tau)=-V(\theta,-\Delta \tau),
$$

which implies $V(\theta, 0)=0$. In effect, if $\tau_{+1}=\tau_{-1}$, the function $\eta(t)$ given by (26) satisfies the time-shift symmetry (11) and consequently the average velocity is zero. Therefore, in the dichotomic case, a necessary condition for a directed kink motion is that the difference between the residence times in each potential state, $\Delta \tau$, has to be nonzero. Furthermore, in the case where net motion exists, the flux can be reversed through the operation $\Delta \tau \rightarrow-\Delta \tau$.

In our numerical simulations, we have first verified that for $\Delta \tau \neq 0$ a nonzero average velocity is observed. In Fig. 3 , the time evolutions of the kink center, $X(t)$, for $\Delta \tau=-2$ (open circles) and $\Delta \tau=0$ (full circles) are compared. Only when the time symmetry (11) is not satisfied by $\eta(t)$ does net motion appear.

In Fig. 4, the average kink velocity computed from simulations (circles) is compared with the average velocity obtained from the collective coordinate theory by using Eq. (21) (solid line). Despite considering small values of $\theta$ and a slow fundamental frequency $\omega=0.1$, the agreement is very poor. The discontinuous character of the dichotomic function $\eta(t)$ is decisive in this poor agreement due to the adiabatic nature of the collective coordinate approximation.

Notice that here the parameter $\theta$ plays a rather different role than in the previous section, since now the potential $U(\Phi, t)$ is $2 \pi$ periodic in $\theta$. The dependence of the kink velocity on the parameter $\theta$ over the whole range $[-\pi, \pi]$ is shown in Fig. 5. The perturbation on the system is very strong for $\theta \gtrsim \pi / 2$. For this reason, it is necessary to apply

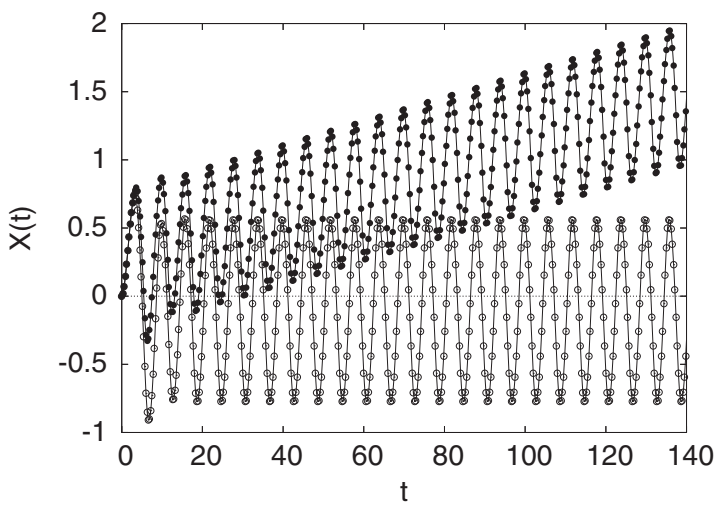

FIG. 3. Time evolution of the kink center with a two-state potential given by (3) and (26). No ratchet effect is observed when $\eta(t)$ satisfies the time-shift symmetry (11) (open circles, $\Delta \tau=0$ ). Net kink motion appears breaking that symmetry by setting $\Delta \tau=-2$ (full circles). The remaining parameters are $\theta=0.8, \omega=\pi / 3$, and $\beta=0.8$.

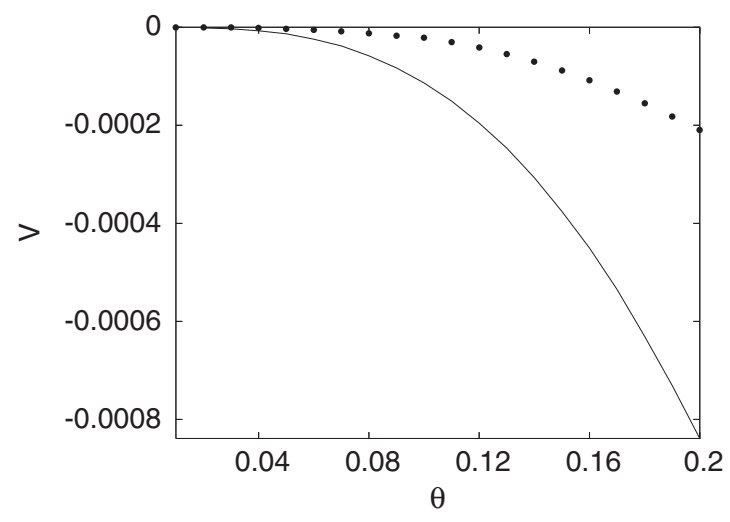

FIG. 4. Comparison of the average kink velocity (circles) with the average velocity obtained using the collective coordinate approximation (solid line) for $\beta=0.05, \Delta \tau=-2$, and $\omega=0.1$.

a sufficiently large dissipation to prevent the kink from being destroyed. The full circles correspond to a damping coefficient $\beta=0.8$, while for the open circles, $\beta=1$. In agreement with our symmetry analysis, it can be clearly appreciated that $V$ is odd in $\theta$ [Eq. (10)]. Furthermore, it is $\pi$ periodic. In order to understand this new symmetry, notice that given a solution $\Phi\left(x, t ; \theta, \Delta \tau, t_{0}\right)$ of the problem defined by Eqs. (2)(7) and (26), then $\pi+\Phi\left(x, t ; \theta-\pi, \Delta \tau, t_{0}\right)$ is also solution of the same problem except for the initial condition (6). Therefore, if it is additionally assumed that the average kink velocity is independent of the initial conditions, we obtain

$$
V(\theta, \Delta \tau)=V(\theta-\pi, \Delta \tau) .
$$

This relation, together with $V(0, \Delta \tau)=0$, also implies

$$
V( \pm \pi, \Delta \tau)=0
$$

for any value of $\Delta \tau$. This property trivially follows from the fact that the two states of the potential $U_{+1}$ and $U_{-1}$ coincide when $\theta=n \pi$, with $n$ being any integer number. Moreover, by setting $\theta=\pi / 2$ in (29) and bearing in mind Eq. (10), it is easy to conclude that

$$
V( \pm \pi / 2, \Delta \tau)=0
$$

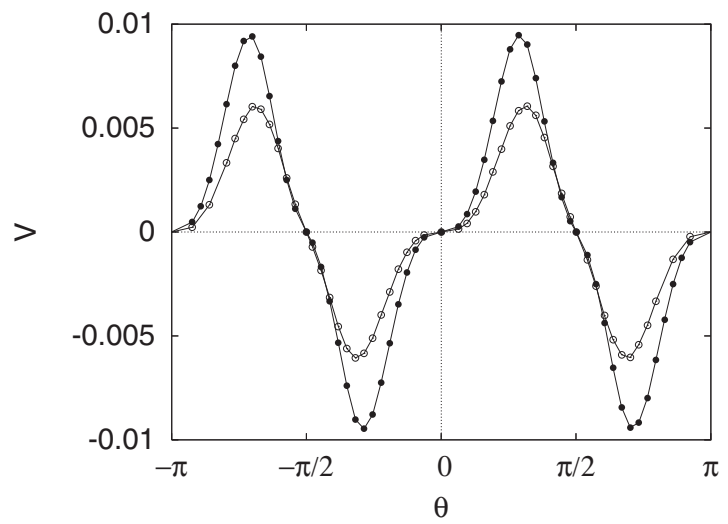

FIG. 5. Kink velocity versus the translation parameter $\theta$ for fixed $\Delta \tau=-2$ and $\omega=\pi / 3$. The open circles correspond to a damping coefficient $\beta=1$, while full circles correspond to $\beta=0.8$. 


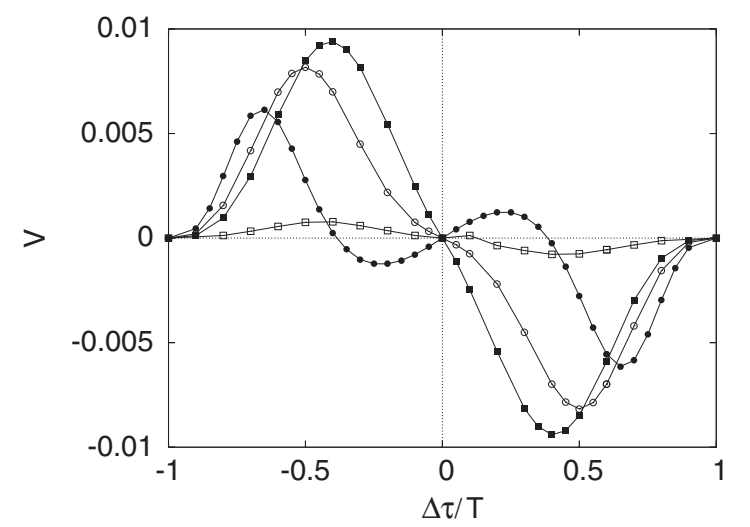

FIG. 6. Kink velocity versus $\Delta \tau / T$ for fixed $T$ and $\theta$. Open squares: $T=4$. Full squares: $T=6$. Open circles: $T=10$. Full circles: $T=14$. In all cases, $\theta=0.8$ and $\beta=0.8$.

The properties (30) and (31) are visible in Fig. 5. The existence of maxima and minima follows directly from the three above equations.

A similar nonmonotonic behavior of the kink velocity is found when it is plotted versus $\Delta \tau / T$ for fixed $T$ and $\theta$, as shown in Fig. 6. The maxima and minima can be easily understood taking into account that no net motion is possible if no temporal symmetry is broken $(\Delta \tau=0)$ and that neither is any net motion possible if no alternation between the potential states occurs $(\Delta \tau / T= \pm 1)$. It should also be borne in mind that there is current reversal due to the symmetry (28).

Figure 6 also provides the intuition that $V$ must display another maximum if it is plotted versus $T$ for fixed $\Delta \tau$. Such behavior is shown in Fig. 7 as a function of the fundamental frequency $\omega=2 \pi / T$. The full and open circles represent results obtained from numerical simulation for $\Delta \tau=-2$ and $\Delta \tau=-0.5$, respectively. On the one hand, in the limit $\omega \rightarrow 0$, the ratchet effect disappears. In this limit, the time intervals $\tau_{+1}$ and $\tau_{-1}$ are much longer than $\beta^{-1}$, which gives roughly the time scale of the relaxation process that takes place each time we switch the potential state. As a consequence, the kink moves at the beginning of the residence times $\tau_{ \pm 1}$, but it

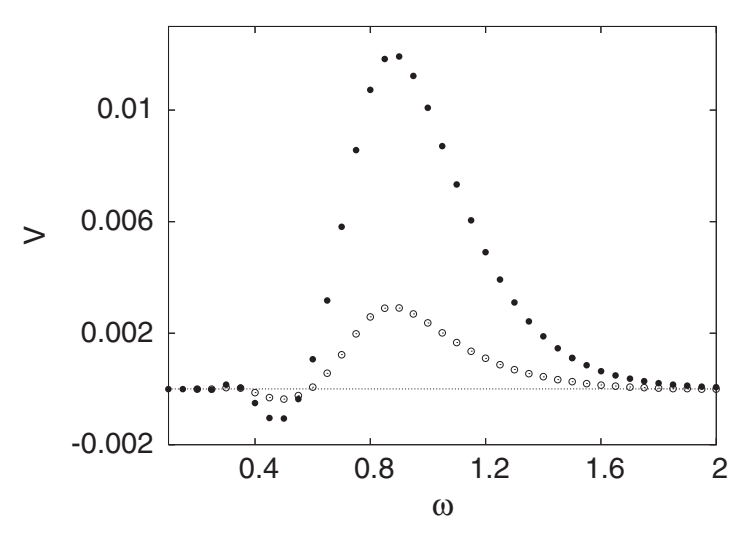

FIG. 7. Kink velocity versus $\omega=2 \pi / T$ for fixed $\Delta \tau$. Full circles correspond to $\Delta \tau=-2$, while open circles correspond to $\Delta \tau=$ -0.5 . The remaining parameters are $\theta=0.8$ and $\beta=0.8$. stops long before those residence times finish. Hence, the distance traveled by a kink in one of the potential states is completely recovered when the potential switches to the other state and, consequently, no net displacement is achieved for each period. On the other hand, neither does the ratchet effect exist when $\omega \rightarrow \infty$. In this case, $\tau_{ \pm 1} \ll \beta^{-1}$, that is, the residence times are so short that the kink is unable to respond to the perturbation. As a result, between these two limits, $V$ has to show at least one maximum or minimum. Additionally, one can observe several current inversions that appear in the low-frequency region.

\section{CONCLUSIONS}

The ratchet dynamics of sine-Gordon kinks induced by phase perturbations has been investigated. Symmetry analysis shows that net motion can be generated when the phase perturbation of the potential, $\theta \eta(t)$, breaks the time-shift symmetry (11). Remarkably, the kink moves with a nonzero average velocity in the absence of any external force and maintaining the field potential symmetry at every time instant.

An approximated theory, with an ansatz with three collective coordinates, namely, the center of the soliton, its width, and the background field, has been developed in order to shed light on the ratchet mechanism of the kink. Using this ansatz, it is assumed that the functional form of the soliton is preserved although the collective coordinates become time dependent. In contrast to other soliton ratchets $[4,7,24,30]$, no ratchet effect is predicted here in absence of the background.

Moreover, when a biharmonic phase perturbation is used, the agreement between the collective coordinate theory and the simulations of the sine-Gordon system is excellent, even for relatively large perturbation amplitudes. However, when a dichotomic perturbation is employed, the agreement is poor, thereby creating the challenge of finding a better theory for discontinuous perturbations.

The dependence of the kink average velocity on the system parameters has been explored in detail. The rich phenomenology observed can be understood through symmetry considerations that allow certain features to be explained, such as the suppression of transport for particular values of the parameters, nonmonotonic behaviors, and current inversions.

Although we specifically investigate the existence of this novel soliton ratchet mechanism within the framework of the sine-Gordon equation, the obtained results can easily be generalized to other models with topological soliton solutions, such as the double sine-Gordon and $\phi^{4}$ systems [30,31].

\section{ACKNOWLEDGMENTS}

We acknowledge financial support from the Ministerio de Ciencia e Innovación of Spain through Grant No. FIS200802873 (B.S.-R. and J.C.-P.), from the Ministerio de Economía y Competitividad of Spain through Grant No. FIS2014-54497-P (N.R.Q.), and from the Junta de Andalucía. N.R.Q. also acknowledges financial support from the Alexander von Humboldt Foundation of Germany through the Research Fellowship for Experienced Researchers SPA No. 1146358 STP and from the Junta de Andalucía through Grant No. P11-FQM-7276. 
[1] O. H. Olsen and M. R. Samuelsen, Sine-Gordon $2 \pi$-kink dynamics in the presence of small perturbations, Phys. Rev. B 28, 210 (1983); M. J. Rice, Physical dynamics of solitons, ibid. 28, 3587 (1983); F. Marchesoni and C. R. Willis, Brownian motion of a sine-Gordon kink, Phys. Rev. A 36, 4559 (1987).

[2] A. Sánchez and A. R. Bishop, Collective coordinates and lengthscale competition in spatially inhomogeneous soliton-bearing equations, SIAM Rev. 40, 579 (1998).

[3] P. Reimann, Brownian motors: Noisy transport far from equilibrium, Phys. Rep. 361, 57 (2002); P. Hänggi and R. Bartussek, Brownian rectifiers: How to convert Brownian motion into directed transport, Lect. Notes Phys. 476, 294 (1996); R. D. Astumian and P. Hänggi, Brownian motors, Phys. Today 55(11), 33 (2002).

[4] M. Salerno and Y. Zolotaryuk, Soliton ratchetlike dynamics by ac forces with harmonic mixing, Phys. Rev. E 65, 056603 (2002).

[5] F. Marchesoni, Thermal Ratchets in 1+1 Dimensions, Phys. Rev. Lett. 77, 2364 (1996); M. Salerno and N. R. Quintero, Soliton ratchets, Phys. Rev. E 65, 025602 (2002).

[6] G. Costantini, F. Marchesoni, and M. Borromeo, String ratchets: ac driven asymmetric kinks, Phys. Rev. E 65, 051103 (2002).

[7] L. Morales-Molina, N. R. Quintero, F. G. Mertens, and A. Sánchez, Internal Mode Mechanism for Collective Energy Transport in Extended Systems, Phys. Rev. Lett. 91, 234102 (2003).

[8] N. R. Quintero, B. Sánchez-Rey, and M. Salerno, Analytical approach to soliton ratchets in asymmetric potentials, Phys. Rev. E 72, 016610 (2005).

[9] F. Jülicher, A. Ajdari, and J. Prost, Modeling molecular motors, Rev. Mod. Phys. 69, 1269 (1997); H. Linke, Ratchets and Brownian motors: Basics, experiments and applications, Appl. Phys. A, 75, 167 (2002); J. S. Bader, R. W. Hammond, S. A. Henck, M. W. Deem, G. A. McDermott, J. M. Bustillo, J. W. Simpson, G. T. Mulhern, and J. M. Rothberg, DNA transport by a micromachined Brownian ratchet device, Proc. Natl. Acad. Sci. USA 96, 13165 (1999).

[10] P. Hänggi and F. Marchesoni, Artificial Brownian motors: Controlling transport on the nanoscale, Rev. Mod. Phys. 81, 387 (2009); C. S. Lee, B. Jankó, I. Derényi, and A. L. Barabási, Reducing vortex density in superconductors using the "ratchet effect", Nature (London) 400, 337 (1999); J. E. Villegas, S. Savel'ev, F. Nori, E. M. González, J. V. Anguita, R. García, and J. L. Vicent, A superconducting reversible rectifier that controls the motion of magnetic flux quanta, Science 302, 1188 (2003).

[11] A. V. Ustinov, C. Coqui, A. Kemp, Y. Zolotaryuk, and M. Salerno, Ratchetlike Dynamics of Fluxons in Annular Josephson Junctions Driven by Biharmonic Microwave Fields, Phys. Rev. Lett. 93, 087001 (2004).

[12] E. Goldobin, H. Susanto, D. Koelle, R. Kleiner, and S. A. van Gils, Oscillatory eigenmodes and stability of one and two arbitrary fractional vortices in long Josepson $0-\kappa$ junctions, Phys. Rev. B 71, 104518 (2005).

[13] S. Ooi, S. Savel'ev, M. B. Gaifullin, T. Mochiku, K. Hirata, and F. Nori, Nonlinear Nanodevices Using Magnetic Flux Quanta, Phys. Rev. Lett. 99, 207003 (2007).

[14] M. Beck, E. Goldobin, M. Neuhaus, M. Siegel, R. Kleiner, and D. Koelle, High-Efficiency Deterministic Josephson Vortex Ratchet, Phys. Rev. Lett. 95, 090603 (2005).

[15] G. Carapella and G. Costabile, Ratchet Effect: Demonstration of a Relativistic Fluxon Diode, Phys. Rev. Lett. 87, 077002 (2001).
[16] L. Morales-Molina, F. G. Mertens, and A. Sánchez, Soliton ratchets out of point-like inhomogeneities, Eur. Phys. J. B 37, 79 (2004).

[17] L. Morales-Molina, F. G. Mertens, and A. Sánchez, Ratchet behavior in nonlinear Klein-Gordon systems with pointlike inhomogeneities, Phys. Rev. E 72, 016612 (2005).

[18] A. L. Sukstanskii and K. I. Primak, Kink Drift Motion in the $\phi^{4}$ Model, Phys. Rev. Lett. 75, 3029 (1995); Yu. S. Kivshar and A. Sánchez, Kink Drift in Oscillating Fields, ibid. 77, 582 (1996).

[19] S.-H. Lee and D. G. Grier, Flux reversal in a two-state symmetric optical thermal ratchet, Phys. Rev. E 71, 060102 (2005); J. Casado-Pascual, Flux reversal in a simple random-walk model on a fluctuating symmetric lattice, ibid. 74, 021112 (2006); M. Zelan, H. Hagman, G. Labaigt, S. Jonsell, and C. M. Dion, Experimental measurement of efficiency and transport coherence of a cold-atom Brownian motor in optical lattices, ibid. 83, 020102 (2011).

[20] E. Goldobin, A. Sterck, T. Gaber, D. Koelle, and R. Kleiner, Dynamics of Semifluxons in Nb Long Josephson 0- $\pi$ Junctions, Phys. Rev. Lett. 92, 057005 (2004); H. Susanto, Localized modes and phonon scattering of a lattice $\kappa$ kink, Phys. Rev. E 73, 026608 (2006).

[21] Q. Liang, Y. Yu, Q. Wang, and J. Dong, Controllable 0- $\pi$ Transition in a Superconducting Graphene-Nanoribbon Junction, Phys. Rev. Lett. 101, 187002 (2008).

[22] V. V. Ryazanov, V. A. Oboznov, A. Yu. Rusanov, A. V. Veretennikov, A. A. Golubov, and J. Aarts, Coupling of Two Superconductors Through a Ferromagnet: Evidence for a $\pi$ Junction, Phys. Rev. Lett. 86, 2427 (2001).

[23] N. R. Quintero, B. Sánchez-Rey, and J. Casado-Pascual, Comment on Soliton ratchets induced by excitation of internal modes, Phys. Rev. E 71, 058601 (2005).

[24] V. Stehr, P. Müller, F. G. Mertens, and A. Bishop, Soliton ratchets in sine-Gordon systems with additive inhomogeneities, Phys. Rev. E 79, 036601 (2009).

[25] D. Poletti, T. J. Alexander, E. A. Ostrovskaya, B. Li, and Yu. S. Kivshar, Dynamics of Matter-Wave Solitons in a Ratchet Potential, Phys. Rev. Lett. 101, 150403 (2008).

[26] V. Berardi, J. Lydon, P. G. Kevrekidis, C. Daraio, and R. Carretero-González, Directed ratchet transport in granular chains, Phys. Rev. E 88, 052202 (2013).

[27] J. A. Cuesta, N. R. Quintero, and R. Alvarez-Nodarse, Time Invariance Determines the Functional Shape of the Current in Rocking Ratchets, Phys. Rev. X 3, 041014 (2013)

[28] J. Casado-Pascual, J. A. Cuesta, N. R. Quintero, and R. AlvarezNordarse, General approach for dealing with dynamical systems with spatiotemporal periodicities, Phys. Rev. E 91, 022905 (2015).

[29] S. Pagano, M. Salerno, and M. R. Samuelsen, Parametric adiabatic perturbations on the sine-Gordon breather solution, Physica D 26, 396 (1987).

[30] L. Morales-Molina, N. R. Quintero, A. Sánchez, and F. G. Mertens, Soliton ratchets in homogeneous nonlinear KleinGordon systems, Chaos 16, 013117 (2006).

[31] N. R. Quintero, R. Alvarez-Nodarse, and F. G. Mertens, Driven and damped double sine-Gordon equation: The influence of internal modes on the soliton ratchet mobility, Phys. Rev. E 80, 016605 (2009). 